\title{
Signal-to-noise ratio uniformity and stability of agar gel phantom with iron (III) oxide as relaxation modifier
}

\author{
W. X. Er ${ }^{1}$, W. J. Lim ${ }^{1}$, Y. Dwihapsari ${ }^{2}$, M. N. A. Awang ${ }^{3}$ and A. N. Yusoff ${ }^{4^{*}}$ (1)
}

\begin{abstract}
Background: Agar has been commonly used as one of the materials to fabricate magnetic resonance imaging phantoms in the past few decades. In this study, eleven agar gel phantoms with different iron (III) oxide $\left(\mathrm{Fe}_{2} \mathrm{O}_{3}\right)$ masses were prepared. This study was aimed to evaluate the signal-to-noise ratio (SNR) uniformity and stability of agar gel phantoms with and without the addition of $\mathrm{Fe}_{2} \mathrm{O}_{3}$ at two different time points (TPs). $\mathrm{Fe}_{2} \mathrm{O}_{3}$ powder was used as a relaxation modifier to manipulate and produce various SNR, T1 and T2 values. These phantoms were scanned using turbo spin echo pulse sequence to produce T1- and T2-measurement images. The SNR was then computed by plotting 1, 3 and 25 regions of interest on the images using ImageJ software. The T1 and T2 relaxation equations were then fitted to the experimental results of SNR versus TR and SNR versus TE curves for the determination of saturation $\left(\mathrm{SNR} \mathrm{R}_{\mathrm{o}}\right), \mathrm{T} 1$ and $\mathrm{T} 2$ values.

Results: The results demonstrated that the agar gel phantoms were able to maintain SNR uniformity but not SNR stability after 4 weeks of phantom preparation. The change in the water content and microstructure of the phantoms have no significant effect on $\mathrm{T} 2$ relaxation but on $\mathrm{T} 1$ relaxation. The $\mathrm{T} 1$ and $\mathrm{T} 2$ of the agar gel phantoms were minimally affected although there was a systemic increase in the content of the $\mathrm{Fe}_{2} \mathrm{O}_{3}$ powder.

Conclusions: It can be concluded that the agar gel phantoms exhibited the characteristics of SNR uniformity, but they showed instability of SNR at TP2. The $\mathrm{Fe}_{2} \mathrm{O}_{3}$ in powder form is not an effective relaxation modifier to reduce the $\mathrm{T} 1$ and $\mathrm{T} 2$ when it is introduced into the agar gel phantoms. Dissolved nanosized particles should be the focus of future studies.
\end{abstract}

Keywords: Agar gel, Signal-to-noise ratio, Uniformity, Stability, Iron (III) oxide, Relaxation time

\section{Background}

Nowadays, magnetic resonance imaging (MRI) phantoms are designed to possess the properties of human tissues. Human tissue mimicking materials are gaining increasing attention in this field of research. The materials used to fabricate an MRI phantom should be easy to handle, cost-effective, robust, non-hazardous and stable [1]. In

\footnotetext{
*Correspondence: nazlimtrw@ukm.edu.my

${ }^{4}$ Center for Diagnostic, Therapeutic and Investigative Studies, Faculty of Health Science, Universiti Kebangsaan Malaysia, Jalan Raja Muda Abdul Aziz, 50300 Kuala Lumpur, Malaysia

Full list of author information is available at the end of the article
}

previous studies, agarose, agar, polyvinyl alcohol (PVA), carrageenan gel and water have been used to produce phantoms [1]. Water phantom is easy to handle but it is also affected by vibration, producing air bubbles that can cause non-uniformity. Another concern when using a water phantom is the relaxation time of water that is much longer than human tissue. Hence, other materials that have relaxation times closer to human tissue are gaining more attention [1-6]. A phantom made from human tissue mimicking material is reliable as its T1 and $\mathrm{T} 2$ fall in a range possesses by most human tissues, which makes the experimental result closer to the result performed on human subjects [7]. This is an advantage as 
the human subject is not required in the research and the accuracy of the result is guaranteed.

Furthermore, MRI phantom has developed a new role in the standardization protocol of quantitative MRI (qMRI) biomarkers. This is also the key to the success of clinical trials in integrating qMRI biomarkers as primary endpoints. In testing the accuracy, repeatability and reproducibility of qMRI biomarkers, the use of phantom is crucial. Basic imaging parameters, such as SNR and relaxation times, should be acquired from the MRI phantom and established as part of the quality assurance program for qMRI [8]. Therefore, it is crucial to obtain the practical T1 and T2 for agar to investigate its suitability as a phantom material [3].

In this study, agar gel phantoms were developed to fulfill the objective of this study which was to test the uniformity and stability of SNR for investigating the suitability of the material as an MRI phantom. Agar is an organic product derived from red algae and seaweeds, such as Gracilaria and Gelidium, and is a mixture of two components including a linear polysaccharide (agarose) and a heterogeneous mixture of small molecules known as agaro-pectin. It is a biological gelling agent that is nontoxic, cost-effective, easy to make and use and less prone to leakage. Although various types of phantom materials, such as polyvinyl alcohol (PVA), gelatin, carrageenan and distilled water, have been proposed, agar and agarose gel are the most commonly used materials to fabricate the MRI phantom with the addition of relaxation modifier as agar and agarose have fulfilled the desired characteristics of MRI phantoms $[1-3,5,6]$.

As agar is a hydrogel and consists of water molecules, it can be used in diffusion MRI measurement to observe the diffusion pattern of water molecules in the agar [2]. Agarose gel phantoms also aid in MRI-based radiotherapy treatment planning by providing more accurate quantitative features of disease and healthy tissue rather than depending solely on qualitative assessment [9]. Furthermore, as the resting-state fMRI depends on transversal relaxation of tissue (T2*), the usage of agar as phantom materials can result in local inhomogeneity of the magnetic field which will further reduce the $\mathrm{T} 2$ * value. The effect is more pronounced when the tumormimicking materials have been introduced in the agar gel phantoms [6].

To modify the relaxation time of the agar gel phantoms, a relaxation modifier known as contrast agent must be added. Theoretically, it can shorten the relaxation time of protons in water molecules by increasing the efficiency of energy transfer in $\mathrm{T} 1$ relaxation and dephasing of proton in T2 relaxation [10]. Since the first study of contrastenhanced MRI with the use of ferric chloride as a contrast agent in 1981, the use of contrast-enhanced study in clinical studies has been widely used to date [11]. Superparamagnetic $\mathrm{Fe}_{2} \mathrm{O}_{3}$ nanoparticle is one of the examples of negative contrast agents. It can also be a T1 contrast agent by modifying the particle size and coating which are still under research for several years [11, 12]. In previous studies, different concentrations of gadolinium (III) oxide have been used as relaxation modifier in agarose gel or PVA slime phantoms to obtain the T1 and T2 values $[5,13]$.

In this research, different masses of superparamagnetic $\mathrm{Fe}_{2} \mathrm{O}_{3}$ in powder form were added into the agar gel phantoms to investigate its effect on both $\mathrm{T} 1$ and $\mathrm{T} 2$ values. To the best of our knowledge, several studies have quantified T1 or T2 values or merely the SNR values of agar gel phantoms with different types of relaxation modifiers through various protocols such as standard, diffusion MRI and fMRI protocols. Therefore, this research aimed to compare the image quality of agar gel MRI phantom with different $\mathrm{Fe}_{2} \mathrm{O}_{3}$ masses at 1,3 and 25 regions-ofinterest (ROIs) at different time points (TPs). SNR represents the signal-to-noise ratio obtained from each agar gel phantom directly from the equation. Each phantom has its own specific $S N R$ value. $\mathrm{SNR}_{\mathrm{o}}$ is the $\mathrm{SNR}$ acquired when the longitudinal magnetization $\left(\mathrm{M}_{\mathrm{z}}\right)$ of the proton spins has returned to its equilibrium state, which means when the saturation of proton spins has been achieved. These were obtained after plotting the $\mathrm{T} 1$ and $\mathrm{T} 2$ curves by the curve fitting tool in MATLAB ${ }^{\circledR}$. SNR was used to compare the SNR uniformity between 1, 3 and 25 ROIs, while $\mathrm{SNR}_{\mathrm{o}}$ was used to compare the stability of SNR between two different TPs. The SNR uniformity and stability of the agar gel phantoms obtained in this study can be assured as this method can improve the precision of SNR measurement by using 1,3 and 25 ROIs $[5,13-15]$.

\section{Methods}

This study was conducted according to the guidelines for ethical reviews and approved by the Institutional Ethics Committee (IEC).

The materials and apparatus required for the preparation of agar gel phantom were as follows: (1) agar powder $\geq 99.65 \%$ (Sigma Aldrich, Malaysia); (2) relaxation modifier, iron (III) oxide $\left(\mathrm{Fe}_{2} \mathrm{O}_{3}\right)$ 85.9\%; (3) distilled water; (4) sterile plastic container; (5) digital weighing scale; (6) conical flask; (7) duct tape; (8) magnetic stirrer hotplate.

The agar gel phantoms were prepared in a biomedical laboratory. The agar gel phantoms were then scanned using the SIEMENS Magnetom Verio 3-T MRI machine.

Eleven agar gel phantoms with different $\mathrm{Fe}_{2} \mathrm{O}_{3}$ masses $(0 \mathrm{~g}, 0.002 \mathrm{~g}, 0.004 \mathrm{~g}, 0.006 \mathrm{~g}, 0.008 \mathrm{~g}, 0.010 \mathrm{~g}, 0.012 \mathrm{~g}$, $0.014 \mathrm{~g}, 0.016 \mathrm{~g}, 0.018 \mathrm{~g}$ and $0.020 \mathrm{~g}$ ) were prepared using conventional mixing method. One distilled water 
phantom was also prepared as control. For each agar phantom, $2.55 \mathrm{~g}$ of agar powder was mixed with $85 \mathrm{ml}$ of distilled water at room temperature. Magnetic stirring technique was used for $8 \mathrm{~min}$ to achieve a homogeneous distribution of agar in the distilled water while the solution was heated to $90{ }^{\circ} \mathrm{C}$ on the hot plate. The change in color of the mixture from cloudy to clear indicated that the solution was in a completely uniform distribution state. Then, the $\mathrm{Fe}_{2} \mathrm{O}_{3}$ powder was gently mixed and stirred in the agar mixture for another $2 \mathrm{~min}$. The agar mixture with $\mathrm{Fe}_{2} \mathrm{O}_{3}$ powder was poured into an $85-\mathrm{ml}$ sterilized plastic container (see Additional file 1: Appendix Figure A1) and allowed to cool gradually to room temperature after the lid was tightly sealed. The phantoms were kept in an air-tight desiccator before the scanning day. The prepared phantoms were scanned at two different TPs, namely 2 weeks (TP1) and 4 weeks (TP2) after the preparation. Table 1 shows the volume of distilled water, mass of agar powder, concentration of agar mixture and mass of $\mathrm{Fe}_{2} \mathrm{O}_{3}$ powder used in phantom preparation.

Data acquisition was performed by scanning all the agar gel phantoms using a 3-T SIEMENS Magnetom Verio MRI machine. Before and until the day of scanning, the condition of the agar gel phantoms was daily examined to ensure absence of air bubbles and fungal growth as these would impact the image quality. On the MRI couch, eleven agar gel phantoms and one distilled water phantom were positioned horizontally at the iso-center of the MRI bore inside the head coil with a laser aligned at the center of both the phantoms and the head coil as shown in Additional file 1: Appendix Figure A2 and A3. This was to ensure that all the phantoms were longitudinally subjected to the same magnitude of magnetic field strength during scanning. The middle slice of all the phantoms was obtained to calculate the SNR value. Turbo spin echo (TSE) sequence was applied to obtain both the T1- and T2-measurement images in axial projection. Except for TR and TE, other imaging parameters were set as constant, which included slice thickness of $10.0 \mathrm{~mm}$, field of view (FOV) of $200 \times 200 \mathrm{~mm}$ and a matrix size of $256 \times 256$. The phase encoding direction for both T1- and T2-measurement images was set from right to left. For T1-measurement image, the TR was set from 400 to $8000 \mathrm{~ms}$ with a constant TE value of $18 \mathrm{~ms}$ as short TE was needed to acquire T1-measurement image. For T2-measurement image, the TE was set from 12 to $233 \mathrm{~ms}$ with a constant TR of $2000 \mathrm{~ms}$ as long TR was needed to acquire T2-measurement image. All images acquired were stored in a CD-ROM in DICOM format. The phantoms were scanned at two respective TP1 and TP2 to test the stability of the phantoms. Examples of images obtained from T1- and T2-measurements are shown in Additional file 1: Appendix Figure A4 and A5.

Image software (The National Institute of Health, $\mathrm{NIH}$ ) and MATLAB ${ }^{\circledR}$ (The Math Works, Inc., Natick) were used in the data analysis, while the Statistical Packages for Social Sciences (SPSS version 25) was used to assess the uniformity and stability of agar gel phantoms with different $\mathrm{Fe}_{2} \mathrm{O}_{3}$ concentrations. After the images were obtained, the ImageJ software was used to specify the ROIs on the middle axial slice of the obtained images to calculate the SNR, T1 and T2 values. First, the SNR determination was performed by placing the 1, 3 and 25 ROIs on images of the twelve phantoms accordingly to measure the signal intensities. For the placement of $1 \mathrm{ROI}$, the size of the ROI was made up to $1005 \mathrm{~mm}^{2}$ on each phantom image. For 3 and 25 ROIs, they were

Table 1 The compositions of agar gel phantoms

\begin{tabular}{|c|c|c|c|c|}
\hline Phantom & Volume of distilled water (ml) & Mass of agar powder (g) & $\begin{array}{l}\text { Concentration of agar solution } \\
\left(\mathrm{gml}^{-1}\right)\end{array}$ & $\begin{array}{l}\text { Mass of } \\
\mathrm{Fe}_{2} \mathrm{O}_{3} \\
\text { powder }(\mathrm{g})\end{array}$ \\
\hline 1 & 85 & 2.55 & 0.03 & 0 \\
\hline 2 & 85 & 2.55 & 0.03 & 0.002 \\
\hline 3 & 85 & 2.55 & 0.03 & 0.004 \\
\hline 4 & 85 & 2.55 & 0.03 & 0.006 \\
\hline 5 & 85 & 2.55 & 0.03 & 0.008 \\
\hline 6 & 85 & 2.55 & 0.03 & 0.010 \\
\hline 7 & 85 & 2.55 & 0.03 & 0.012 \\
\hline 8 & 85 & 2.55 & 0.03 & 0.014 \\
\hline 9 & 85 & 2.55 & 0.03 & 0.016 \\
\hline 10 & 85 & 2.55 & 0.03 & 0.018 \\
\hline 11 & 85 & 2.55 & 0.03 & 0.020 \\
\hline Water & 85 & 0.00 & 0.00 & 0.000 \\
\hline
\end{tabular}


randomly placed on the phantom images and the respective ROI sizes were 'large' and 'XXL' in the ImageJ setting. The signal intensity $\left(I_{\mathrm{p}}\right)$ for 1 ROI was entirely taken from the ROI, while for the 3 and 25 ROIs the mean $I_{\mathrm{p}}$ was obtained over the ROIs. Another ROI was then specified as large as possible on the background of all the images to obtain noise intensity $\left(I_{\mathrm{b}}\right)$ and its standard deviation $\left(\sigma_{\mathrm{b}}\right)$. The noise intensities were averaged for all images to obtain the mean noise intensity and standard deviation. The SNR determination was performed on both the T1 (various TR and fixed TE) and T2 (various TE and fixed TR) measurement images using Eq. (1).

$$
\mathrm{SNR}=\left(I_{\mathrm{p}}-I_{\mathrm{b}}\right) / \sigma_{\mathrm{b}}
$$

The $\mathrm{T} 1$ and $\mathrm{T} 2$ values of the phantoms were determined based on the SNR against TR plot (T1 curve) from the equation of $\mathrm{SNR}(\mathrm{TR})=\mathrm{SNR}_{\mathrm{o}}\left(1-e^{-\mathrm{TR} / \mathrm{T1} 1}\right)$ and SNR against TE plot (T2 curve) using the equation of $\mathrm{SNR}(\mathrm{TE})=\mathrm{SNR}_{i}\left(e^{-\mathrm{TE} / \mathrm{T} 2}\right)$, respectively. The value of $\mathrm{SNR}_{\mathrm{o}}$ was obtained at long $\mathrm{TR}$, while the value of $\mathrm{SNR}_{\mathrm{i}}$ was obtained at the beginning of $\mathrm{T} 2$ relaxation. $\mathrm{SNR}_{\mathrm{o}}$ is defined as the SNR obtained when the longitudinal magnetization $\left(M_{\mathrm{z}}\right)$ of proton spins has reached an equilibrium. The experimental data were fitted to both equations using MATLAB ${ }^{\circledR}$ R2019b (The Math Works, Inc., Natick) curve fitting toolbox. The T1 and T2 values were obtained upon achieving the minimum sum of squared difference between the observed and fitted data. By referring to the fitted $\mathrm{T} 1$ and $\mathrm{T} 2$ curves, the saturation of SNR $\left(\mathrm{SNR}_{\mathrm{o}}\right)$ and the initial maximum SNR for T2 curve $\left(\mathrm{SNR}_{\mathrm{i}}\right)$ were also estimated. The $95 \%$ confidence interval (CI) standard sum of errors (SSE), root mean square errors (RMSE) and adjusted and unadjusted $R^{2}$ of fit were also computed. The $R^{2}$ of fit is the correlation coefficient between the observed and fitted data and the ideal value of $R^{2}$ is 1 . The $R^{2}$ values of all agar gel phantoms were averaged and recorded. The T1 and T2 curves in this study were fitted in such a way that the $R^{2}$ of fit was maximized and the SSE was minimized. The SSE obtained was then converted to standard deviation (SD) using Eq. (2) from which $n$ is the number of phantoms. The effects of different $\mathrm{Fe}_{2} \mathrm{O}_{3}$ content and different $\mathrm{TP}$ on $R^{2}, \mathrm{SNR}_{\mathrm{o}}, \mathrm{SNR}_{\mathrm{i}}, \mathrm{T} 1$ and $\mathrm{T} 2$ were evaluated and discussed. The recorded quantities were also compared between 1,3 and 25 ROIs at each TP.

$$
\mathrm{SD}=\sqrt{\mathrm{SSE} /(n-1)}
$$

\section{Results}

3.1 Effects of different number of ROIs on SNR uniformity

Figures 1 and 2 show the T1 and T2 curves of all agar gel and distilled water phantoms obtained from 1, 3 and 25
ROIs at two different TPs. As expected, the T1 curves for all agar gel and distilled water phantoms exhibit an exponentially increasing trend as TR is increased while TE is fixed at $18 \mathrm{~ms}$ with no indication of saturation. The T2 curves for all agar gel phantoms exhibit an exponentially decreasing trend as TE is increased while TR is fixed at $2000 \mathrm{~ms}$, while for distilled water phantom, the trend is different from the phantoms but consistent among all ROIs and at both TPs. It can be summarized that all agar gel phantoms show similar trend of $\mathrm{T} 1$ and $\mathrm{T} 2$ relaxations for all ROIs and at both TP1 and TP2 as indicated by the overlapping of the T1 and T2 curves. Distilled water phantom, however, shows a distinct $\mathrm{T} 1$ and $\mathrm{T} 2$ curves as compared to those of the agar gel phantoms for all ROIs and at both TP1 and TP2. For T1 curves, the distinction between distilled water phantom and agar gel phantoms is larger when comparison is made between TP1 and TP2 from which distilled water phantom shows higher SNR at higher TR for TP2 for all ROIs. For T2 curves, the distinction between distilled water phantom and agar gel phantoms is obvious with a longer relaxation time and higher SNR for distilled water phantom. This occurs for all ROIs and at both TP1 and TP2. There is a difference in T1 SNR obtained using different numbers of ROIs between TP1 and TP2 from which the values at TP2 are relatively higher. T2 SNR for all ROIs however, remains equal at both TPs.

SNR uniformity can be investigated by comparing the $R^{2}$ values between different number of ROIs at any one TP and between TP for each of the ROI. The T1 and T2 $R^{2}$ data obtained from all ROIs at both TPs are shown in Tables 3, 4 and 5 .

Kruskal-Wallis test conducted to examine the difference in the $R^{2}$ values of all agar gel between the number of ROIs at TP1 revealed no significant difference in $R^{2}$ values between 1, 3 and 25 ROIs for both T1 $R^{2}$ and T2 $R^{2}$ at a significant level of $0.05\left(p=0.732\right.$ for T1 $R^{2}$ and $p=0.331$ for $\mathrm{T} 2 R^{2}$ ). Insignificant difference in $R^{2}$ values (correlation coefficient between the observed and fitted data or the goodness of fit) between 1, 3 and 25 ROIs indicated uniformity of the SNR of the prepared agar gel phantoms.

On the contrary, Kruskal-Wallis test on the $R^{2}$ values at TP2 showed a significant difference in at least one pair among 1,3 and 25 ROIs $\left(p<0.001\right.$ for T $1 R^{2}$ and $p=0.002$ for T2 $R^{2}$ ) for T1 and T2 $R^{2}$ at a significant level of 0.05 . Tukey post hoc test indicated that the T1 $R^{2}$ values differ significantly between 1 and 3 ROI and between 1 and 25 ROI $(p<0.05)$, while for T2 $R^{2}$ values, significant difference was observed between 1 and 3 ROI and between 3 and 25 ROI $(p<0.05)$.

The results of Mann-Whitney U test comparisons between the two TPs revealed that T1 $R^{2}$ for 1 ROI 

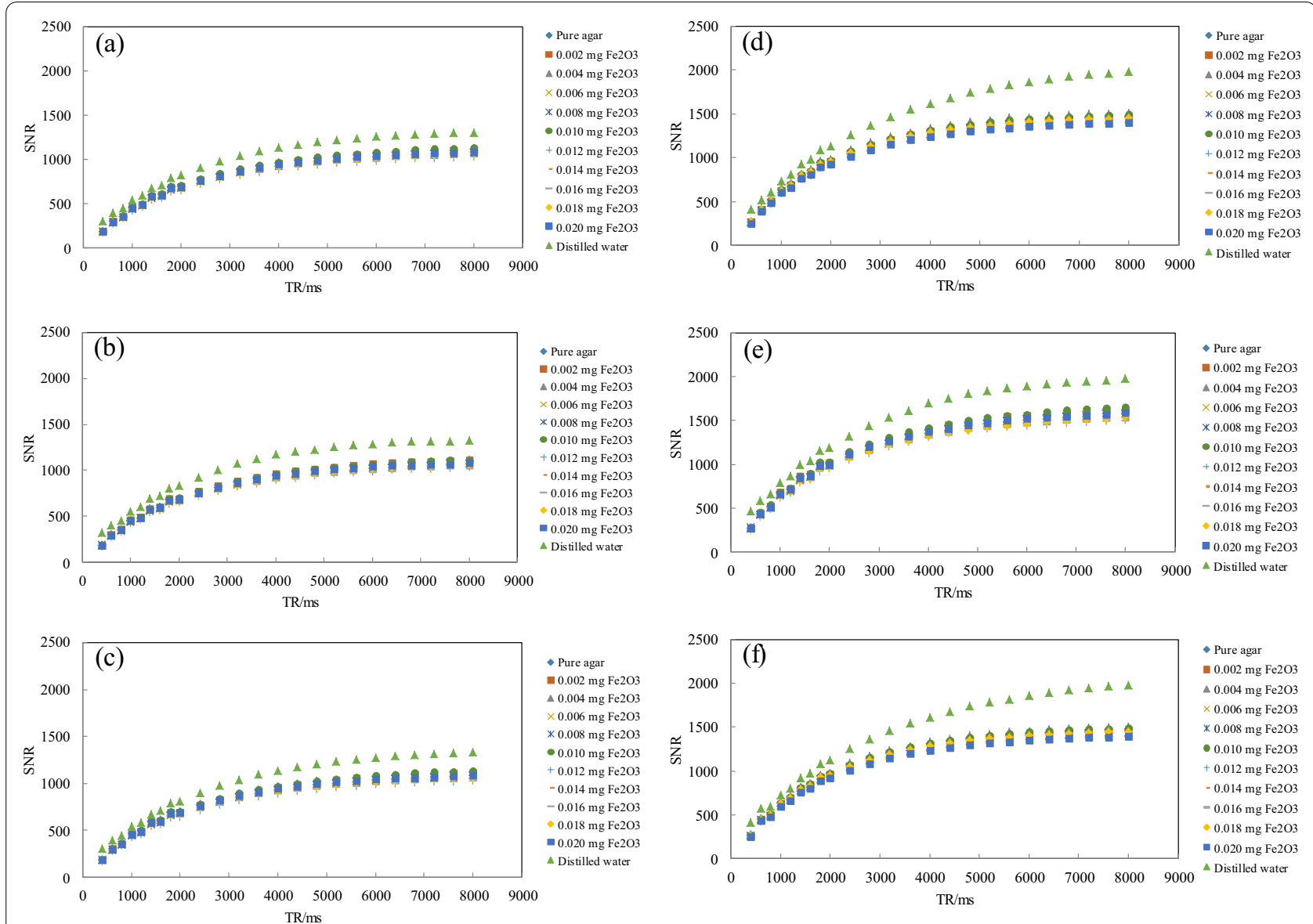

Fig. 1 T1 curves obtained from the phantoms at TP1 (left) by using a 1 ROI, b 3 ROIs and $\mathbf{c} 25$ ROls, and at TP2 (right) by using $\mathbf{d} 1$ ROI, e 3 ROIs and f $25 \mathrm{ROls}$

at TP2 is significantly higher than at TP1 $(p<0.001)$. A significantly higher T1 $R^{2}$ was also obtained for 25 ROI at TP2 $(p<0.05)$ as compared to TP1. However, no significant difference was observed in T1 $R^{2}$ for 3 ROI between TP1 and TP2 $(p>0.05)$. For T2 $R^{2}$, comparisons between TP1 and TP2 for 1,3 and 25 ROIs revealed no significant difference $(p>0.05)$.

The statistical analysis results above showed that there was a significant data dispersion when different numbers of ROI were used to obtain SNR value at TP2 possibly originating from loss of uniformity. However, the $R^{2}$ values of all T1 and T2 SNR curves for 1,3 and 25 ROIs were close to 1 , meaning that even though the data dispersion between the simulated and experimental SNR were significantly different, the difference is small.

The results also revealed that the SNR uniformity degradation over time was only observed on the T1 curves. No significant change in uniformity is observed on T2 curves.

\subsection{Effects of TP on SNR stability of agar gel phantoms}

SNR stability over time can be concluded by comparing the $\mathrm{SNR}_{\mathrm{o}}$ and $\mathrm{SNR}_{\mathrm{i}}$ values between different number of ROIs at any one TP and between TP for each of the ROI. The T1 and T2 SNR data obtained from all ROIs at both TPs are shown in Tables 3, 4 and 5. At TP1 (Tables 2, 4), it can be seen that the value of $\mathrm{SNR}_{\mathrm{o}}$ for each agar gel phantom is about the same for 1,3 and 25 ROIs. However, the value of $\mathrm{SNR}_{\mathrm{i}}$ for each agar gel phantom is relatively higher for 3 ROIs as compared to the other two ROIs. Kruskal-Wallis test conducted to examine the difference in the $\mathrm{SNR}_{\mathrm{o}}$ values of all agar gel between the number of ROIs reveals no significant difference in $\mathrm{SNR}_{\mathrm{o}}$ values between 1, 3 and 25 ROIs for T1 $\operatorname{SNR}_{\mathrm{o}}(p=0.957)$ at a significant level of 0.05 . Similar insignificant differences were obtained for $\mathrm{T}_{2} \operatorname{SNR}_{\mathrm{i}}(p=0.063)$.

At TP2 (Tables 3, 5), the values of $\mathrm{SNR}_{\mathrm{o}}$ and $\mathrm{SNR}_{\mathrm{i}}$ for all agar gel phantoms obtained from 3 ROIs are relatively higher than 1 and 25 ROIs. Kruskal-Wallis test on the $\mathrm{SNR}_{\mathrm{o}}$ values showed a significant difference in at least 

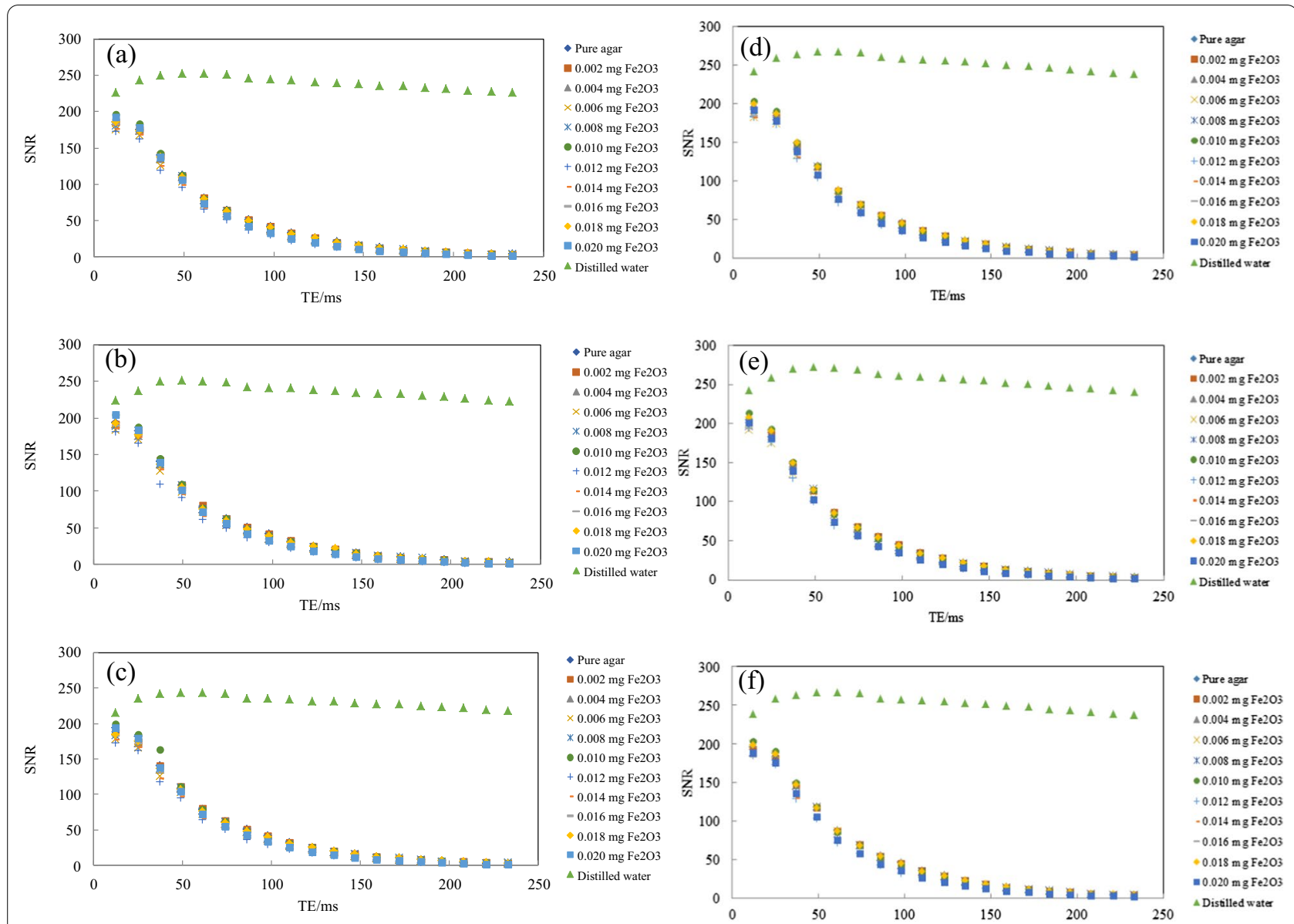

Fig. 2 T2 curves obtained from the phantoms at TP1 (left) by using a 1 ROI, b 3 ROls and $\mathbf{c} 25$ ROls, and at TP2 (right) by using $\mathbf{d} 1$ ROI, e 3 ROls and f $25 \mathrm{ROIs}$
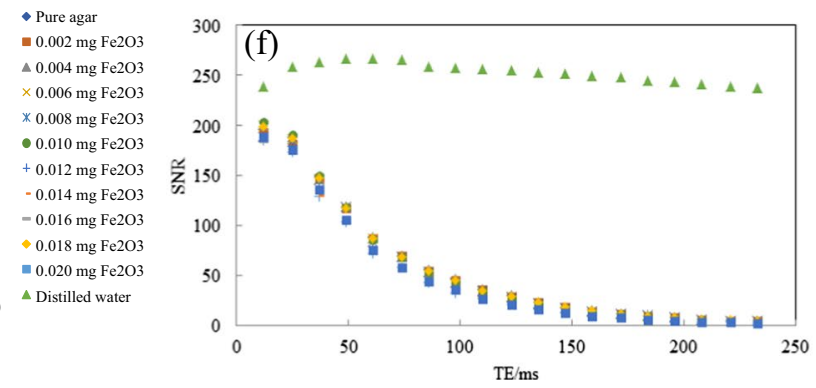

- Pure agar

$=0.002 \mathrm{mg} \mathrm{Fe} 203$ $\triangle 0.004 \mathrm{mg}$ Fe203 $\times 0.006 \mathrm{mg} \mathrm{Fe} 203$ $x 0.008 \mathrm{mg} \mathrm{Fe} 203$ $-0.010 \mathrm{mg} \mathrm{Fe} 203$ $+0.012 \mathrm{mg} \mathrm{Fe} 203$ $-0.014 \mathrm{mg} \mathrm{Fe} 203$ $-0.016 \mathrm{mg} \mathrm{Fe} 203$ $-0.018 \mathrm{mg} \mathrm{Fe} 203$ $=0.020 \mathrm{mg} \mathrm{Fe} 203$ Disilled water

Table $2 \mathrm{SNR}_{\mathrm{o}}$ and $R^{2}$ values of agar gel and water phantoms for T1 curves at TP1

\begin{tabular}{|c|c|c|c|c|c|c|}
\hline \multirow[t]{3}{*}{ Phantom } & \multicolumn{6}{|l|}{ TP1 } \\
\hline & \multicolumn{3}{|l|}{$\mathrm{SNR}_{\mathrm{o}}$} & \multicolumn{3}{|l|}{$R^{2}$} \\
\hline & $1 \mathrm{ROI}$ & $3 \mathrm{ROI}$ & $25 \mathrm{ROI}$ & $1 \mathrm{ROI}$ & $3 \mathrm{ROI}$ & $25 \mathrm{ROI}$ \\
\hline 1 & 1116 & 1106 & 1120 & 0.99790 & 0.99769 & 0.99790 \\
\hline 2 & 1112 & 1117 & 1113 & 0.99789 & 0.99766 & 0.99797 \\
\hline 3 & 1129 & 1116 & 1131 & 0.99864 & 0.99862 & 0.99867 \\
\hline 4 & 1093 & 1094 & 1093 & 0.99891 & 0.99890 & 0.99877 \\
\hline 5 & 1081 & 1073 & 1084 & 0.99868 & 0.99861 & 0.99866 \\
\hline 6 & 1137 & 1120 & 1136 & 0.99810 & 0.99813 & 0.99808 \\
\hline 7 & 1038 & 1041 & 1037 & 0.99886 & 0.99886 & 0.99882 \\
\hline 8 & 1046 & 1052 & 1044 & 0.99869 & 0.99856 & 0.99868 \\
\hline 9 & 1037 & 1025 & 1034 & 0.99802 & 0.99781 & 0.99797 \\
\hline 10 & 1064 & 1062 & 1065 & 0.99817 & 0.99793 & 0.99833 \\
\hline 11 & 1091 & 1091 & 1092 & 0.99834 & 0.99850 & 0.99841 \\
\hline Water & 1251 & 1286 & 1276 & 0.99944 & 0.99878 & 0.99951 \\
\hline
\end{tabular}


Table $3 \mathrm{SNR}_{\mathrm{o}}$ and $R^{2}$ values of agar gel and water phantoms for T1 curves at TP2

\begin{tabular}{|c|c|c|c|c|c|c|}
\hline \multirow[t]{3}{*}{ Phantom } & \multicolumn{6}{|l|}{ TP2 } \\
\hline & \multicolumn{3}{|l|}{$\mathrm{SNR}_{\mathrm{o}}$} & \multicolumn{3}{|l|}{$R^{2}$} \\
\hline & $1 \mathrm{ROI}$ & 3 ROls & 25 ROls & $1 \mathrm{ROI}$ & 3 ROls & $25 \mathrm{ROls}$ \\
\hline 1 & 1494 & 1626 & 1474 & 0.99928 & 0.99792 & 0.99869 \\
\hline 2 & 1484 & 1628 & 1465 & 0.99934 & 0.99788 & 0.99878 \\
\hline 3 & 1530 & 1628 & 1508 & 0.99934 & 0.99858 & 0.99880 \\
\hline 4 & 1453 & 1601 & 1435 & 0.99957 & 0.99884 & 0.99913 \\
\hline 5 & 1468 & 1567 & 1448 & 0.99940 & 0.99839 & 0.99875 \\
\hline 6 & 1511 & 1652 & 1494 & 0.99933 & 0.99797 & 0.99876 \\
\hline 7 & 1424 & 1517 & 1412 & 0.99957 & 0.99898 & 0.99901 \\
\hline 8 & 1413 & 1536 & 1398 & 0.99959 & 0.99887 & 0.99912 \\
\hline 9 & 1390 & 1509 & 1364 & 0.99942 & 0.99789 & 0.99882 \\
\hline 10 & 1468 & 1535 & 1449 & 0.99933 & 0.99804 & 0.99875 \\
\hline 11 & 1417 & 1592 & 1396 & 0.99937 & 0.99835 & 0.99881 \\
\hline Water & 1957 & 1900 & 1942 & 0.99971 & 0.99912 & 0.99919 \\
\hline
\end{tabular}

Table $4 \mathrm{SNR}_{\mathrm{i}}$ and $R^{2}$ values of agar gel and water phantoms for T2 curves at TP1

\begin{tabular}{|c|c|c|c|c|c|c|}
\hline \multirow[t]{3}{*}{ Phantom } & \multicolumn{6}{|l|}{ TP1 } \\
\hline & \multicolumn{3}{|l|}{$\mathrm{SNR}_{\mathrm{i}}$} & \multicolumn{3}{|l|}{$R^{2}$} \\
\hline & $1 \mathrm{ROI}$ & 3 ROIs & $25 \mathrm{ROls}$ & $1 \mathrm{ROI}$ & 3 ROls & $25 \mathrm{ROIs}$ \\
\hline 1 & 250.7 & 261.2 & 261.2 & 0.98960 & 0.98960 & 0.98960 \\
\hline 2 & 250.7 & 259.1 & 251.4 & 0.98910 & 0.98920 & 0.99040 \\
\hline 3 & 253.6 & 260.2 & 255.2 & 0.99120 & 0.99330 & 0.99160 \\
\hline 4 & 240.3 & 250.5 & 241.9 & 0.99830 & 0.99830 & 0.98950 \\
\hline 5 & 248.3 & 258.6 & 252.0 & 0.98510 & 0.98510 & 0.98480 \\
\hline 6 & 265.8 & 276.0 & 274.8 & 0.99010 & 0.99010 & 0.98110 \\
\hline 7 & 239.6 & 249.2 & 238.5 & 0.98740 & 0.98740 & 0.98810 \\
\hline 8 & 246.3 & 259.5 & 246.3 & 0.98580 & 0.98580 & 0.98810 \\
\hline 9 & 255.9 & 265.5 & 256.6 & 0.98460 & 0.98460 & 0.98550 \\
\hline 10 & 250.3 & 257.8 & 249.5 & 0.99010 & 0.99010 & 0.98880 \\
\hline 11 & 266.4 & 283.0 & 269.6 & 0.98840 & 0.98840 & 0.98830 \\
\hline Water & 287.1 & 466.7 & 173.6 & 0.98530 & 0.98500 & 0.97340 \\
\hline
\end{tabular}

one pair among 1, 3 and 25 ROIs $(p<0.001)$ for T1 SNR at a significant level of 0.05 . Tukey post hoc test conducted showed that there was no significant difference in $\mathrm{SNR}_{\mathrm{o}}$ between 1 and 3 ROIs $(p=1.000)$. However, there exists significant differences in $\mathrm{SNR}_{\mathrm{o}}$ between 1 and 25 ROIs $(p=0.001)$ and between 3 and 25 ROIs $(p<0.001)$ at a significant level of 0.05 .

Kruskal-Wallis test on the $\mathrm{SNR}_{\mathrm{i}}$ values showed a significant difference in at least one pair among 1,3 and 25 ROIs $(p<0.001)$ for $\mathrm{T} 2 \mathrm{SNR}_{\mathrm{i}}$ at a significant level of 0.05 . Tukey post hoc test conducted showed that there are no significant differences in $\mathrm{SNR}_{\mathrm{i}}$ between 1 and 25 ROIs $(p=1.000)$ at a significant level of 0.05 . However, comparisons showed significant differences in $\mathrm{SNR}_{\mathrm{i}}$ between 1 and 3 ROIs $(p=0.006)$ and between 3 and 25 ROIs $(p=0.013)$ at a significant level of 0.05 . Higher $\mathrm{SNR}_{\mathrm{i}}$ can be seen for 3 ROIs as compared to 1 ROI and 25 ROIs. The results from Mann-Whitney $U$ test indicated that $\mathrm{SNR}_{\mathrm{o}}$ values for $\mathrm{T} 1$ curves and $\mathrm{SNR}_{\mathrm{i}}$ values for $\mathrm{T} 2$ curves increase significantly $(p<0.001)$ at a significant level of 0.05 from TP1 to TP2 for all 1, 3 and 25 ROIs, with the exception of comparison between $\mathrm{SNR}_{\mathrm{i}}$ for 25 ROIs which is not significant $(p<0.05)$. Looking at a small change in $\mathrm{SNR}_{\mathrm{o}}$ and $\mathrm{SNR}_{\mathrm{i}}$ shown in Tables 2, 3, 4 and 5 when $\mathrm{Fe}_{2} \mathrm{O}_{3}$ is systematically added to the pure agar gel, it can be said that the addition of $\mathrm{Fe}_{2} \mathrm{O}_{3}$ is seen to have little 
Table $5 \mathrm{SNR}_{\mathrm{i}}$ and $R^{2}$ values of agar gel and water phantoms for T2 curves at TP2

\begin{tabular}{|c|c|c|c|c|c|c|}
\hline \multirow[t]{3}{*}{ Phantom } & \multicolumn{6}{|l|}{ TP2 } \\
\hline & \multicolumn{3}{|l|}{$\mathrm{SNR}_{\mathrm{i}}$} & \multicolumn{3}{|l|}{$R^{2}$} \\
\hline & $1 \mathrm{ROI}$ & 3 ROls & $25 \mathrm{ROIs}$ & $1 \mathrm{ROI}$ & 3 ROls & $25 \mathrm{ROls}$ \\
\hline 1 & 261.0 & 271.7 & 262.1 & 0.99030 & 0.99240 & 0.99080 \\
\hline 2 & 258.3 & 269.8 & 257.9 & 0.98950 & 0.99160 & 0.99030 \\
\hline 3 & 268.3 & 279.0 & 267.2 & 0.99060 & 0.99330 & 0.99110 \\
\hline 4 & 245.8 & 258.7 & 267.2 & 0.98780 & 0.99260 & 0.99110 \\
\hline 5 & 256.0 & 266.1 & 255.9 & 0.98640 & 0.98820 & 0.98660 \\
\hline 6 & 272.9 & 285.6 & 273.7 & 0.99050 & 0.99290 & 0.99030 \\
\hline 7 & 251.0 & 265.5 & 253.3 & 0.98830 & 0.99120 & 0.98910 \\
\hline 8 & 250.9 & 272.4 & 253.7 & 0.98710 & 0.99150 & 0.98880 \\
\hline 9 & 256.3 & 269.9 & 255.4 & 0.98560 & 0.98800 & 0.98620 \\
\hline 10 & 266.7 & 276.9 & 266.0 & 0.98930 & 0.99110 & 0.98990 \\
\hline 11 & 262.7 & 275.6 & 258.2 & 0.98910 & 0.99120 & 0.98910 \\
\hline Water & 136.8 & 133.2 & 144.4 & 0.98530 & 0.98810 & 0.98690 \\
\hline
\end{tabular}

Table 6 T1 values of all agar gel phantoms at TP1

\begin{tabular}{|c|c|c|c|c|c|}
\hline \multirow[t]{2}{*}{ Phantom } & \multirow{2}{*}{$\begin{array}{l}1 \mathrm{ROI} \\
\mathrm{T} 1 \text { (ms) }\end{array}$} & \multicolumn{2}{|l|}{3 ROls } & \multicolumn{2}{|l|}{25 ROls } \\
\hline & & $\mathrm{T} 1$ (ms) & SD & $\mathrm{T} 1$ (ms) & SD \\
\hline 1 & 2093 & 2103 & 14.35 & 2094 & 13.84 \\
\hline 2 & 2090 & 2085 & 14.55 & 2088 & 13.53 \\
\hline 3 & 2095 & 2093 & 11.17 & 2090 & 11.11 \\
\hline 4 & 2090 & 2082 & 9.78 & 2087 & 10.31 \\
\hline 5 & 2157 & 2119 & 10.81 & 2152 & 10.72 \\
\hline 6 & 2101 & 2076 & 13.04 & 2107 & 13.42 \\
\hline 7 & 2041 & 2054 & 9.44 & 2040 & 9.54 \\
\hline 8 & 2019 & 2019 & 10.70 & 2022 & 10.17 \\
\hline 9 & 2027 & 2030 & 12.86 & 2031 & 12.50 \\
\hline 10 & 2034 & 2061 & 12.99 & 2001 & 11.63 \\
\hline 11 & 2006 & 2009 & 11.30 & 2014 & 11.65 \\
\hline Water & 2287 & 2262 & 12.26 & 2464 & 7.78 \\
\hline
\end{tabular}

effects on the magnetization of all agar gel phantoms in this study.

\subsection{Effect of $\mathrm{Fe}_{2} \mathrm{O}_{3}$ addition on $\mathrm{T} 1$ and $\mathrm{T} 2$ of agar gel phantoms}

As shown in Figs. 1 and 2, the T1 and T2 curves for all the agar gel phantoms demonstrate similar trend for all $\mathrm{Fe}_{2} \mathrm{O}_{3}$ contents, from which T1 SNR increases exponentially as TR is increased when TE was fixed at $18 \mathrm{~ms}$, and T2 SNR decreases exponentially as TE is increased when TR was fixed at $2000 \mathrm{~ms}$. As a result, the T1 and T2 of agar gel phantoms with different $\mathrm{Fe}_{2} \mathrm{O}_{3}$ content are about the same when observed for each ROI at both TP but differ markedly with that of distilled water (see Tables 6, 7,
Table 7 T1 values of all agar gel phantoms at TP2

\begin{tabular}{|c|c|c|c|c|c|}
\hline \multirow[t]{2}{*}{ Phantom } & \multirow{2}{*}{$\begin{array}{l}1 \mathrm{ROI} \\
\mathrm{T} 1 \text { (ms) }\end{array}$} & \multicolumn{2}{|l|}{3 ROls } & \multicolumn{2}{|l|}{25 ROls } \\
\hline & & $\mathrm{T} 1$ (ms) & SD & $\mathrm{T1}$ (ms) & SD \\
\hline 1 & 1886 & 2087 & 19.99 & 1920 & 14.17 \\
\hline 2 & 1892 & 2085 & 20.20 & 1928 & 13.61 \\
\hline 3 & 1900 & 2106 & 16.53 & 1932 & 13.86 \\
\hline 4 & 1898 & 2087 & 14.72 & 1923 & 11.23 \\
\hline 5 & 1904 & 2121 & 16.98 & 1928 & 13.61 \\
\hline 6 & 1898 & 2093 & 20.06 & 1920 & 13.96 \\
\hline 7 & 1879 & 2038 & 13.00 & 1913 & 11.74 \\
\hline 8 & 1862 & 2027 & 13.87 & 1886 & 10.94 \\
\hline 9 & 1883 & 2024 & 18.57 & 1915 & 12.44 \\
\hline 10 & 1873 & 2037 & 18.23 & 1901 & 13.55 \\
\hline 11 & 1849 & 2015 & 17.33 & 1882 & 12.73 \\
\hline Water & 2837 & 2516 & 15.46 & 2953 & 15.17 \\
\hline
\end{tabular}

8 , 9). T1 and T2 values for distilled water are much longer than for any agar gel phantom.

Kruskal-Wallis test conducted to compare the T1 values for all agar gel phantoms between 1, 3 and 25 ROIs at TP1 revealed no significant difference $(p>0.05)$ at a significant level of 0.05 .

At TP2, significant differences in T1 values existed among 1,3 and 25 ROIs $(p<0.001)$ at a significant level of 0.05 . Tukey post hoc test results showed that there was no significant difference in $\mathrm{T} 1$ values between 1 and 25 ROIs pair $(p=0.114)$. Significant differences in T1 values were, however, observed between 1 and 3 ROIs $(p<0.001)$ and between 3 and 25 ROIs $(p=0.009)$ pairs at a significant level of 0.05 . 
Table 8 T2 values of all agar gel phantoms at TP1

\begin{tabular}{|c|c|c|c|c|c|}
\hline \multirow[t]{2}{*}{ Phantom } & \multirow{2}{*}{$\begin{array}{l}1 \mathrm{ROI} \\
\mathrm{T} 2(\mathrm{~ms})\end{array}$} & \multicolumn{2}{|l|}{3 ROls } & \multicolumn{2}{|l|}{25 ROls } \\
\hline & & $\mathrm{T} 2$ (ms) & SD & $\mathrm{T} 2$ (ms) & SD \\
\hline 1 & 59.91 & 56.19 & 6.20 & 56.19 & 6.20 \\
\hline 2 & 60.07 & 57.69 & 6.32 & 59.60 & 5.82 \\
\hline 3 & 57.04 & 54.37 & 5.91 & 55.84 & 5.44 \\
\hline 4 & 56.25 & 53.17 & 5.64 & 55.81 & 5.78 \\
\hline 5 & 58.91 & 55.90 & 7.04 & 57.57 & 7.29 \\
\hline 6 & 56.13 & 52.90 & 6.11 & 55.76 & 6.35 \\
\hline 7 & 52.18 & 47.92 & 5.71 & 51.86 & 5.95 \\
\hline 8 & 52.59 & 49.82 & 6.79 & 51.71 & 6.16 \\
\hline 9 & 53.12 & 50.56 & 7.09 & 51.96 & 7.19 \\
\hline 10 & 58.59 & 54.93 & 5.64 & 57.48 & 6.20 \\
\hline 11 & 52.88 & 49.12 & 6.12 & 51.66 & 7.04 \\
\hline Water & 1825.00 & 3026.00 & 1.14 & 1115.00 & 1.15 \\
\hline
\end{tabular}

Table 9 T2 values of all agar gel phantoms at TP2

\begin{tabular}{|c|c|c|c|c|c|}
\hline \multirow[t]{2}{*}{ Phantom } & \multirow{2}{*}{$\begin{array}{l}1 \mathrm{ROI} \\
\mathrm{T} 2(\mathrm{~ms})\end{array}$} & \multicolumn{2}{|l|}{3 ROls } & \multicolumn{2}{|l|}{$25 \mathrm{ROIs}$} \\
\hline & & $\mathrm{T} 2$ (ms) & SD & $\mathrm{T} 2(\mathrm{~ms})$ & SD \\
\hline 1 & 61.64 & 57.56 & 5.53 & 61.39 & 5.97 \\
\hline 2 & 62.27 & 58.76 & 5.76 & 62.00 & 6.04 \\
\hline 3 & 59.58 & 55.46 & 5.30 & 59.10 & 5.93 \\
\hline 4 & 57.96 & 54.97 & 5.15 & 59.10 & 5.93 \\
\hline 5 & 61.44 & 57.94 & 6.78 & 61.22 & 7.20 \\
\hline 6 & 58.51 & 54.20 & 5.54 & 57.83 & 6.32 \\
\hline 7 & 54.46 & 50.53 & 5.65 & 53.60 & 6.11 \\
\hline 8 & 54.67 & 50.18 & 5.68 & 53.38 & 6.18 \\
\hline 9 & 54.61 & 51.16 & 6.75 & 54.16 & 7.01 \\
\hline 10 & 60.11 & 56.44 & 5.73 & 59.47 & 6.32 \\
\hline 11 & 54.43 & 50.78 & 5.85 & 54.65 & 6.24 \\
\hline Water & 686.20 & 629.20 & 0.93 & 749.80 & 0.93 \\
\hline
\end{tabular}

Kruskal-Wallis test conducted to compare the T2 values for all agar gel phantoms between 1, 3 and 25 ROIs at TP1 reveals no significant difference $(p=0.090)$ at a significant level of 0.05 .

At TP2, significant differences in T2 values existed among 1,3 and 25 ROIs $(p=0.037)$ at a significant level of 0.05 . Tukey post hoc test results showed that there were no significant differences in $\mathrm{T} 2$ values between 1 and 25 ROIs pair $(p=1.000)$ and between 3 and 25 ROIs pair $(p=0.157)$. A significant difference in T2 values was, however, observed between 1 and 3 ROIs $(p=0.046)$ at a significant level of 0.05 .

Mann-Whitney $U$ test conducted to compare the T1 values for 1, 3 and 25 ROIs between TP1 and TP2 showed that T1 TP1 1 ROI was significantly higher than T1 TP2 1 ROI $(p<0.001)$. Similarly, T1 TP1 25 ROIs were significantly higher than T1 TP2 25 ROIs $(p=0.001)$. However, no significant difference was observed between T1 TP1 3 ROIs and T1 TP2 3 ROIs $(p=0.842)$. There was no significant difference in T2 values between TP1 and TP2 for all 1, 3 and 25 ROIs $(p>0.05)$.

\section{Discussion}

In this study, SNR measurement via ROI analysis was implemented. This method is quite common in acquiring SNR value from an image. In addition, this method is easy and provides a faster way to calculate the regional statistics of the SNR which can be obtained directly from the image $[16,17]$. In this work, the SNR measurement method was refined using 1, 3 and 25 ROIs.

Distilled water has a distinct characteristic of $\mathrm{T} 1$ and T2 curves as compared to the pure agar gel and agar gels containing the relaxation modifier. Higher SNR for distilled water at any TR and TE values (see Figs. 1, 2) is attributed to a higher water content. The same argument can be used to explain a higher $\mathrm{SNR}_{\mathrm{o}}$ for distilled water tabulated in Tables 2 and 3.

Two weeks after preparation, the agar gels almost contain as much water molecules as the distilled water resulting in a small difference between them as shown by their T1 curves at TP1 (Fig. 1-top three). Over time, water content in the agar gels at TP2 decreases mainly due to evaporation, hence a decrease in intensity. The evaporation of water did occur for distilled water but the effects on SNR is more prominent for agar gel phantoms. Hence, the difference in the T1 curves of the agar gels with distilled water becomes clearer at TP2 (Fig. 1-bottom three).

In relation to the above discussion, the intensity of background noise was found to be relatively higher for the agar gels at TP1, resulting in lower SNR for distilled water and agar gels according to Eq. (1), while for the scans conducted 4 weeks after preparation (at TP2), lower background noise intensity resulted in higher SNR. The average background noise intensity and standard deviation of noise for T1 measurements are 15.083 and 2.144 at TP1. The values decrease to 12.461 and 1.466 at TP2. It can be said that a higher background noise at TP1 supersede the decrease in signal intensity at TP2, resulting in an overall higher SNR at TP2 as can be seen when comparing TP1 and TP2 on Fig. 1 (see also Eq. (1)).

For T2 curves (Fig. 2), the average background noise intensity and standard deviation of noise are 10.762 and 7.864 at TP1. At TP2, the values are 9.333 and 6.895 . The small differences between these TP1 and TP2 values explain why the T2 curves are similar regardless of at which TP they were obtained. It can also be said that 
T2 relaxation for all agar gel phantoms and distilled water was not influenced by the water content which was assumed to be lesser at TP2.

The value of $R^{2}$, also known as correlation coefficient, or the goodness of fit measures how disperse are the experimental SNR data about the fitted curve. The agar gel phantoms produce good T1 and T2 SNR uniformity at TP1 across 1, 3 and 25 ROIs which is mainly attributed to the freshness of the phantoms. Water content in all phantoms was at its initial maximum with only little being evaporated, hence no change in SNR across the image. Thus, the choice of number of ROIs is independent of SNR uniformity.

As more and more water evaporated from the phantoms' matrix during the interval between TP1 and TP2, the signal (and noise) across the image starts to fluctuate and resulting in SNR non-uniformity. The $R^{2}$ is no longer indistinguishable between number of ROIs with the exception between 3 and 25 ROIs for T1 SNR and between 1 and 25 ROIs for T2 SNR. It is thought that these ROIs will also show differences in $R^{2}$ if a longer interval is implemented.

The results also show that signal uniformity across an image was not influenced by $\mathrm{Fe}_{2} \mathrm{O}_{3}$ content. Nevertheless, the presence of water in the agar gel phantoms in maintaining the uniformity of signal intensity across an image was vital. It was found that the phantoms were only useful if used immediately after preparation up to 2 weeks. Improvement on the ability of the plastic container in preventing water evaporation such as the formation of water vapor inside the container is crucial and deserves special attention in future studies.

Microstructure changes that occurred during the interval between TP1 and TP2 have been proven to be the cause of significant different in T1 $R^{2}$ for 1 ROI and 25 ROIs. T1 relaxation is microstructure-dependent, also known as spin-lattice relaxation. The loss of an amount of water at TP2 had caused the T1 relaxation data (T1 SNR) to disperse significantly, thus, causing the $R^{2}$ value at TP2 to be distinguishable (significantly higher) to that at TP1 for 1 ROI and 25 ROIs. It is believed that if the interval is longer, the difference in $R^{2}$ between TP1 and TP2 for 3 ROIs may also be noticeable. It is most unlikely that $\mathrm{Fe}_{2} \mathrm{O}_{3}$ was the cause of the increase of dispersion at TP2 because the content of $\mathrm{Fe}_{2} \mathrm{O}_{3}$ remained unchanged at both TPs.

Equal dispersion of T2 SNR between TP1 and TP2 for all the 1, 3 and 25 ROIs clearly indicated that the T2 relaxation mechanism was not influenced by the microstructure changes that occurred within the phantom particularly on the reduction of water content during the interval between TP1 and TP2 as discussed above. $\mathrm{T} 2$ relaxation originates from spin-spin relaxation in the presence of external and local magnetic field inhomogeneities. The fact that at either TP1 or TP2 all the prepared phantoms showed similar $\mathrm{T} 2$ relaxation regardless of the differences in water and $\mathrm{Fe}_{2} \mathrm{O}_{3}$ content obviously discounted the dependence of the T2 relaxation on water and $\mathrm{Fe}_{2} \mathrm{O}_{3}$ Content. Furthermore, under the TSE scheme, contribution from external magnetic field inhomogeneity was nullified by the delivery of multiple RF $180^{\circ}$-pulse leaving only local magnetic fields as the source of inhomogeneity from which the latter had caused $R^{2}$ to be incomparable between TP1 and TP2 for all ROIs.

The uniformity of $\mathrm{SNR}_{\mathrm{o}}$ and $\mathrm{SNR}_{\mathrm{i}}$ was achieved at TP1 regardless of the number of ROIs from which they were measured. Similar to the discussion on the effects of ROIs on $R^{2}$, the phantoms prepared 2 weeks before the scan were still fresh and quite uniform in intensity across the image. At TP2, these characteristics were not as that at TP1 as changes in the microstructure of the phantoms occurred during the interval between TP1 and TP2. At $\mathrm{TP} 2$, most comparisons in $\mathrm{SNR}_{\mathrm{o}}$ and $\mathrm{SNR}_{\mathrm{i}}$ between 1,3 and 25 ROIs return a significant difference which explains that uniformity of the signal across the image is no longer preserved.

It is evident that the stability of the $\mathrm{SNR}_{\mathrm{o}}$ and $\mathrm{SNR}_{\mathrm{i}}$ was not achieved for all agar gel phantoms as indicated by a significant increase of the two values at TP2 for all ROIs with the exception of $\mathrm{SNR}_{\mathrm{i}}$ for 25 ROIs that showed equal $\mathrm{SNR}_{\mathrm{i}}$ at both TPs. These findings are consistent with the results for SNR presented above. As discussed earlier, the main cause of SNR increase at TP2 was due to a relatively higher noise at TP1 as compared to TP2. The argument of this sort should also apply to $\mathrm{SNR}_{\mathrm{o}}$ and $\mathrm{SNR}_{\mathrm{i}}$. Nevertheless, in previous studies, the addition of low concentration of paramagnetic ions have been found to show a large reduction in both $\mathrm{SNR}_{\mathrm{o}}$ and $\mathrm{T} 1$ values of the phantoms $[1,13,19-21]$.

The uniformity of the signal intensity of the $\mathrm{T} 1$ and $\mathrm{T} 2$ measurement images at TP1 was also supported by the small differences in the $\mathrm{T} 1$ and $\mathrm{T} 2$ values obtained from 1,3 and 25 ROIs. It can be said that the spin-lattice and spin-spin relaxations that had occurred within the phantoms during the scans have little influence on signal intensity fluctuation across the images. These again can be attributed to the freshness of the phantoms that were prepared 2 weeks before, with initial content of water and microstructure.

The non-uniformity of the signal intensity across the images starts to appear at TP2 at which the comparisons in T1 and T2 values between 1, 3 and 25 ROIs resulted in significant difference for at least 3 out of 6 comparisons for both $\mathrm{T} 1$ and $\mathrm{T} 2$ values with comparisons of $\mathrm{T} 2$ values between the ROIs indicated difference in only 1 comparison. The change in the spin's environment during 
the interval between TP1 and TP2 as discussed above was obviously the reasons for such differences in the comparisons.

Longitudinally, only T1 value was affected by the change in water content and microstructure of the phantoms that had occur during the interval specifically for 1 ROI and 25 ROIs, while T2 value remains unchanged at TP2 for all ROIs. This evident strengthens previous arguments that the change in the water content and microstructure of the phantoms have no significant effect on $\mathrm{T} 2$ relaxation but on $\mathrm{T} 1$ relaxation. In a previous study, the instability in the agar gel phantoms was indeed existed. T1 of the agar gel phantoms showed a slow increase from TP1 to the next [18].

The use of $\mathrm{Fe}_{2} \mathrm{O}_{3}$ in this study was not able to modify the $\mathrm{T} 1$ of the pure agar gel phantom. Furthermore, the $\mathrm{T} 1$ of the agar gel phantoms apparently did not mimic human tissue even with the addition of different $\mathrm{Fe}_{2} \mathrm{O}_{3}$ masses. The closest human tissue mimicked by the agar gel phantoms was blood (1948 ms) which is fluid in nature. Meanwhile, the T2 of the agar gel phantoms would be able to represent and mimic certain tissues or organs in human body, such as muscle $(50 \mathrm{~ms})$, kidneys (56 ms), and breast fat and glandular (53-54 ms) [20]. However, $\mathrm{Fe}_{2} \mathrm{O}_{3}$ could have been a good relaxation modifier if dissolved in hydrochloric acid $(\mathrm{HCl})$ due to the fact that $\mathrm{Fe}_{2} \mathrm{O}_{3}$ is an insoluble salt and can only be dissolved in $\mathrm{HCl}$. The end product of the reaction is iron (III) chloride $\left(\mathrm{FeCl}_{3}\right)$ which is a soluble salt, and has been proven to be a good T1 relaxation modifier [22].

Several studies have demonstrated that the concentration of gelling agent would affect the T1 and T2 of the gel phantoms [19-23]. In those studies, it was found that by increasing the concentration of gelling agents reduced the $\mathrm{T} 1$ of the gel phantoms. This was related to the water content in the phantoms. High concentration of gel phantom indicates high proportion of agar used and less free water in the gel structure. This creates a high barrier for hydrogen spins to move resulting in a shorter T1. It has also been reported that high gel phantom concentrations resulted in low T2 due to its low water content after evaporation, indicating less dipole-dipole interaction for the mechanism of T2 relaxation $[4,19]$. In this study, the concentration of agar gel phantoms was fixed at $0.03 \mathrm{gml}^{-1}$; hence, any change in $\mathrm{T} 1$ and $\mathrm{T} 2$ relaxations within and between TP1 and TP2 was not attributed to the concentration of the gelling agent.

At TP1 and TP2, the T1 curves of all agar gel phantoms showed an exponential increment as TR increased. However, there was a reduction in $\mathrm{T} 1$ in this study which may be due to the water loss that occurred during the interval, increasing the concentration of the agar gel phantoms. $\mathrm{T} 1$ is a measure of the rate of $\mathrm{T} 1$ relaxation process. A longer TR means more time for longitudinal magnetization to recover. T1 relaxation is also termed as spin-lattice relaxation. Proton spins in the agar gel phantoms release energy to the surrounding and then return to the low energy spin-up state resulting in a recovery of longitudinal magnetization $\left(M_{\mathrm{z}}\right)$ to its equilibrium state. The faster the recovery of $M_{\mathrm{z}}$, the shorter the T1. The agar gel phantoms with shorter T1 indicates higher efficiency of proton spins in releasing its energy to the surrounding as compared to distilled water. This means, the precession frequency of proton spins in the agar gel phantoms is closer to the Larmor frequency and much shorter than that of distilled water. The reduction in water content of the phantoms resulted in the spin-lattice relaxation to become more efficient and thus shortened the T1.

When TR was fixed at $2000 \mathrm{~ms}$, the SNR of all the phantoms decreased exponentially as TE increased for all ROIs at both TP. This was due to the progressive dephasing of the spinning dipoles resulting in gradual decrease of transversal magnetization $\left(M_{\mathrm{x}-\mathrm{y}}\right)$ with time. T2 is a measure of the rate of this dephasing process. It determines the rate at which the excited protons are being out of phase with each other. This trend can be observed in all agar gel phantoms with the addition of $\mathrm{Fe}_{2} \mathrm{O}_{3}$ as a relaxation modifier. Pure agar hydrogel and agarose gel phantoms in other studies have exhibited a similar trend of SNR signal reduction as TE increased with T2 values of $116.71 \mathrm{~ms}$ and $150.00 \mathrm{~ms}$, respectively $[5,19]$. The T2 values of the agar gel phantoms in this study are still in the range of T2 for human tissues $(40-150 \mathrm{~ms})$ [1].

The agar gel phantoms consisted of abundance of hydrogen atoms, hence the spins. When larger $\mathrm{Fe}_{2} \mathrm{O}_{3}$ molecules were introduced into the agar gel phantoms, they caused local magnetic field inhomogeneity which then induced a larger difference from the Larmor frequency of the spins [24]. As a result, the T2 of the agar gel phantoms with the addition of $\mathrm{Fe}_{2} \mathrm{O}_{3}$ was shorter as the spins dephased faster. Although the use of $\mathrm{Fe}_{2} \mathrm{O}_{3}$ as a relaxation modifier has been able to reduce the $\mathrm{T} 2$ of the agar gel phantoms, the change was not clearly manifested at both TP1 and TP2. This may be due to the insolubility of $\mathrm{Fe}_{2} \mathrm{O}_{3}$ in the agar gel phantoms or due to the size of the $\mathrm{Fe}_{2} \mathrm{O}_{3}$ particles.

\section{Conclusions}

Agar gel phantoms exhibited the characteristics of SNR uniformity but they showed instability of SNR at TP2. This was due to the evaporation of water molecules inside the agar gel phantoms which has strong influence on the $\mathrm{SNR}_{\mathrm{o}}$ and $\mathrm{T} 1$ values of the agar gel phantoms. It can be concluded that $\mathrm{Fe}_{2} \mathrm{O}_{3}$ in powder form is not an effective relaxation modifier to reduce the $\mathrm{T} 1$ and $\mathrm{T} 2$ when it is introduced into the agar gel phantoms. The T1 
and T2 of the agar gel phantoms were minimally affected although there was a systemic increase in the mass of the $\mathrm{Fe}_{2} \mathrm{O}_{3}$ powder. Limitations of this study include the use of relaxation modifier in powder form instead of the paramagnetic ion typically in aqueous solution. There were sediments of $\mathrm{Fe}_{2} \mathrm{O}_{3}$ powder at the bottom of some agar gel phantoms. For further studies using $\mathrm{Fe}_{2} \mathrm{O}_{3}$ as the relaxation modifier, nanosized particles should be used and the particles should be dissolved properly before being added to the agar gels. Moreover, the scanning TP of this study was 2 weeks and 4 weeks after the preparation of phantoms. For future studies, it is recommended that the phantoms should be scanned as soon as phantom preparation is complete to obtain the original $\mathrm{SNR}_{\mathrm{o}}$ from the MRI phantoms.

\section{Abbreviations}

CD-ROM: Compact disk-read only memory; Cl: Confident interval; DICOM: Digital imaging and communication in medicine; fMRI: Functional magnetic resonance imaging; HCTM: Hospital Canselor Tuanku Muhriz; MRI: Magnetic resonance imaging; $\mathrm{NIH}$ : National Institute of Health; PVA: Poly(vinyl) alcohol; qMRI: Quantitative MRI; RF: Radiofrequency; RMSE: Root mean square error; ROI: Region of interest; SD: Standard deviation; SNR: Signal-to-noise ratio; SNR: SNR saturation; SNR ; SNR initial; SSE: Standard sum of errors; TE: Echo time; TP: Time point; TR: Repetition time; TSE: Turbo spin echo.

\section{Supplementary Information}

The online version contains supplementary material available at https://doi. org/10.1186/s43088-021-00173-0.

Additional file 1. A1. Plastic container filled with agar mixture with $\mathrm{Fe}_{2} \mathrm{O}_{3}$ powder. A2. The front (left) and back (right) view of plastic containers fitted in the phantom holder. A3. Position of the plastic containers and the phantom holder inside the head coil. A4. T1-measurement images of all phantoms acquired with TR of $400 \mathrm{~ms}$ and TE of $18 \mathrm{~ms}$ at TP1 (left) and TP2 (right). A5. T2-measurement images of all phantoms acquired with TR of $2000 \mathrm{~ms}$ and TE of $74 \mathrm{~ms}$ at TP1 (left) and TP2 (right)

\section{Acknowledgements}

The authors would like to express their gratitude to Mr. Sollahuddin Omar for his assistance in the preparation of the agar gel phantoms. The authors would like to thank the Biomedical Science Program for granting permission to use the equipment for the preparation of the agar gel phantoms and the Department of Radiology, Hospital Canselor Tuanku Mukhriz, for the permission to use the MRI scanner.

\section{Authors' contributions}

ANY, WJL and WXE contributed to conceptualization, methodology and formal analysis. WJL provided software and carried out investigation. ANY and YD performed validation. ANY and MNAA provided resources. WXE performed data curation. WJL and WXE performed writing - original draft preparation. ANY and YD contributed to writing - review and editing, visualization and supervision. ANY and MNAA helped with project administration. ANY provided funding. All authors have read and approved that the manuscript is suitable and ready to be sent for publication.

\section{Funding}

This research was funded by the Centre for Research and Instrumentation Management of the Universiti Kebangsaan Malaysia (CRIM-UKM), Grant Number MUTIARA-A163252, for the expenses on rental, transportation, chemicals and consumables.

\section{Availability of data and materials}

Data are contained within the article. The agar gel phantoms are available from the authors.

\section{Declarations}

Ethics approval and consent to participate

This study was conducted according to the guidelines for ethical reviews and approved by the Research Ethics Committee of the Universiti Kebangsaan Malaysia (protocol code JEP-2020-754, 10 December 2020).

\section{Consent for publication}

Not applicable.

\section{Competing interests}

The authors declare no financial and non-financial competing interests.

\section{Author details}

${ }^{1}$ Diagnostic Imaging and Radiotherapy Program, Faculty of Health Science, Universiti Kebangsaan Malaysia, Jalan Raja Muda Abdul Aziz, 50300 Kuala Lumpur, Malaysia. ${ }^{2}$ Department of Physics, Institut Teknologi Sepuluh Nopember Surabaya (ITS), Kampus ITS Sukolilo, Surabaya 60111, Indonesia. ${ }^{3}$ Department of Radiology, Hospital Canselor Tuanku Muhriz (HCTM), Universiti Kebangsaan Malaysia, Jalan Yackob Latif, 56000 Bandar Tun Razak, Kuala Lumpur, Malaysia. ${ }^{4}$ Center for Diagnostic, Therapeutic and Investigative Studies, Faculty of Health Science, Universiti Kebangsaan Malaysia, Jalan Raja Muda Abdul Aziz, 50300 Kuala Lumpur, Malaysia.

Received: 30 June 2021 Accepted: 13 November 2021

Published online: 27 November 2021

\section{References}

1. Hellerbach A, Schuster V, Jansen A, Sommer J (2013) MRI phantoms-are there alternatives to agar? PLoS ONE 8(8):e70343-e70343

2. Dwihapsari Y, Zakiah SN, Darminto, (2019) On the application of diffusion magnetic resonance imaging for study of agarose. IOP Conf Ser Mater Sci Eng 622:012022

3. Keenan KE, Ainslie M, Barker AJ, Boss MA, Cecil KM, Charles C, Chenevert $T L$, Clarke L, Evelhoch JL, Finn P, Gembris D, Gunter JL, Hill DLG, Jack CR Jr, Jackson EF, Liu G, Russek SE, Sharma SD, Steckner M, Stupic KF, Trzasko JD, Yuan C, Zheng J (2018) Quantitative magnetic resonance imaging phantoms: a review and the need for a system phantom. Magn Reson Med 79(1):48-61

4. Yoshimura K, Kato H, Kuroda M, Yoshida A, Hanamoto K, Tanaka A, Tsunoda M, Kanazawa S, Shibuya K, Kawasaki S, Hiraki Y (2003) Development of a tissue-equivalent MRI phantom using carrageenan gel. Magn Reson Med Off J Int Soc Magn Reson Med 50(5):1011-1017

5. Azhar NAA, Tee HS, Yee YY, Awang MNA, Manan HA, Yusoff AN (2020) T1 and $\mathrm{T} 2$ characteristics of agarose gel phantom with different gadolinium oxide concentration as relaxation modifier. Phys Technol Med 1(1):27-37

6. Yusoff AN, Abdul Rashid NS, Usman Ali S (2018) T2* relaxation of agar gel with and without the presence of tumor-like structure as obtained from resting state fMRI sequence protocol. J Phys Conf Ser 1083:012017

7. Rajeshkumar G, Vishnupriyan R, Selvadeepak S (2020) Tissue mimicking material an idealized tissue model for clinical applications: a review. Mater Today Proc 22(4):2696-2703

8. Deng J, Wang Y (2017) Quantitative magnetic resonance imaging biomarkers in oncological clinical trials: current techniques and standardization challenges. Chronic Dis Transl Med 3(1):8-20

9. Dwihapsari Y, Asdiantoro E, Maulidiyah N (2020) On the assessment of image inhomogeneity using $T 2$ magnetic resonance imaging in head phantom for radiotherapy treatment planning: preliminary study. Appl Magn Reson 51(1):59-69

10. Zhang W, Liu L, Chen H, Hu K, Delahunty I, Gao S, Xie J (2018) Surface impact on nanoparticle-based magnetic resonance imaging contrast agents. Theranostics 8(9):2521-2548 
11. Xiao Y-D, Paudel R, Liu J, Ma C, Zhang Z-S, Zhou S-K (2016) MRI contrast agents: classification and application (Review). Int J Mol Med 38(5):1319-1326

12. Bao Y, Sherwood JA, Sun Z (2018) Magnetic iron oxide nanoparticles as $\mathrm{T} 1$ contrast agents for magnetic resonance imaging. J Mater Chem C 6(6):1280-1290

13. Yee YY, Tee HS, Azhar NAA, Abdul Manan H, Awang MNA, Yusoff AN (2019) T1 and T2 characteristics of poly(vinyl) alcohol slime phantom with different relaxation modifier concentrations. Solid State Sci Technol 27(1 \& 2):105-121

14. Tee HS, Yusoff AN, Yee YY, Manan HA, Awang MNA (2020) SNR, T1 and T2 characteristics of poly(vinyl) alcohol (PVA) MRI slime phantom with different PVA/borax ratio. J Phys Conf Ser 1497:012014

15. Yusoff AN, Ding AZ, Azman N, Awang MNA, Abdul Manan H (2020) Homogeneity and stability of poly (vinyl alcohol) slime phantom with different borax concentration. Solid State Sci Technol 27(1 \& 2):51-67

16. Dietrich O, Raya JG, Reeder SB, Reiser MF, Schoenberg SO (2007) Measurement of signal-to-noise ratios in MR images: influence of multichannel coils, parallel imaging, and reconstruction filters. J Magn Reson Imaging 26(2):375-385

17. Firbank MJ, Coulthard A, Harrison RM, Williams ED (1999) A comparison of two methods for measuring the signal to noise ratio on MR images. Phys Med Biol 44(12):N261-N264

18. Blechinger JC, Madsen EL, Frank GR (1988) Tissue-mimicking gelatinagar gels for use in magnetic resonance imaging phantoms. Med Phys 15(4):629-636

19. Dwihapsari Y, Afriana RD (2018) The study of mechanical properties and relaxation time of agar hydrogel for tissue mimicking phantom material in magnetic resonance imaging. IOP Conf Ser Mater Sci Eng 395:012022

20. Hattori K, Ikemoto Y, Takao W, Ohno S, Harimoto T, Kanazawa S, Oita M, Shibuya K, Kuroda M, Kato H (2013) Development of MRI phantom equivalent to human tissues for 3.0-T MRI. Med Phys 40:032303

21. Thangavel K, Saritas E (2017) Aqueous paramagnetic solutions for MRI phantoms at $3 \mathrm{~T}$ : a detailed study on relaxivities. Turk J Electr Eng Comput Sci 25(3):2108-2121

22. Young IR, Clarke GJ, Bailes DR, Pennock JM, Doyle FH, Bydder GM (1981) Enhancement of relaxation rate with paramagnetic contrast agents in NMR imaging. J Comput Tomogr 5(6):543-547

23. Walker P, Lerski RA, Mathur-De Vré R, Binet J, Yane F (1988) Preparation of agarose gels as reference substances for NMR relaxation time measurement: EEC concerted action program. Magn Reson Imaging 6(2):215-222

24. Yusoff AN (2007) Introduction to magnetic resonance imaging (Pengantar Pengimejan Resonans Magnet). UKM Press, Bangi

\section{Publisher's Note}

Springer Nature remains neutral with regard to jurisdictional claims in published maps and institutional affiliations.

\section{Submit your manuscript to a SpringerOpen ${ }^{\circ}$ journal and benefit from:}

- Convenient online submission

- Rigorous peer review

- Open access: articles freely available online

- High visibility within the field

- Retaining the copyright to your article 